\title{
Analytical and Experimental Study of Grinding Forces with Flap Wheels
}

\author{
Alexander N. Unyanin ${ }^{1, a^{*}}$ and Wladimir S. Chistjakov ${ }^{1, b}$ \\ ${ }^{1}$ Faculty of Mechanical Engineering, Ulyanovsk State Technical University, 32, Severny Venetz \\ str., 432027, Ulyanovsk, Russia \\ aa_un@mail.ru, bv_s_chistyakov@mail.ru
}

Keywords: Grinding, Abrasive Grain, Flap Wheel, Force, Workpiece

\begin{abstract}
Analytically, the dependences for calculating the grinding forces with flap wheels are obtained. The forces were defined as the sum of the forces associated with dispersion and friction of the cutting and plastically deforming petal grains against the workpiece. The dependencies take into account the change in the depth of penetration of the grain into the workpiece material along the length of the arc of the contact of the circle with the workpiece. Numerical modeling and experimental study of forces have been carried out. The discrepancy between the calculated and experimental values of the forces does not exceed $20 \%$.
\end{abstract}

\section{Introduction}

Abrasion with flap wheels made of abrasive paper is used to polish and remove the defective layer from the surfaces of the workpieces.

In the process of processing, the petal circle is positioned relative to the workpiece surface to be processed at a distance at which the petals are deformed. The radial force with which the flap wheel acts on the workpiece during processing is made up of centrifugal and elastic components [1]. This force can be varied by changing the speed of the circle and deformation of the petals. The radial force with which the flap wheel acts on the workpiece must be equal to the radial component of the force of dispersion of the workpiece material by the circle. Otherwise, the required rate of material removal from the workpiece will not be provided. Therefore, it is necessary to know the forces arising in the process of grinding with flap wheels.

Mathematical models for calculating grinding forces analytically were developed by S.N. Korchak [2], L.N. Filimonov [3], V.I. Ostrovsky [4], V.V. Efimov [5], J. Peklenik, A. Pahlitzsch and other researchers.

S.N. Korchak [2] established the relationship between the cutting forces of a single abrasive grain (AG) and shear and compression stresses, which depend on the strength of the workpiece material at the deformation rate and temperature in the grinding zone. L.N. Filimonov [3] obtained equations for calculating the cutting forces by a single AG, taking into account the inertial component. This is important for grinding at high wheel speeds, when the inertial component is of great importance. V.V. Efimov [8] switched from calculating cutting forces with a single grain to cutting forces with a grinding wheel. Not only grains producing microcutting are taken into account, but also grains performing plastic deformation. Models of grinding forces, including those with superimposed vibrations, are presented in [6], [7], [8].

The dependencies for calculating the grinding forces with flap wheels are given in [9]. However, they do not take into account the change in the depth of penetration of abrasive grains on the trajectory of their contact with the workpiece and the dispersion of the workpiece by plastically deforming grains. 


\section{Analytical research}

The components of the grinding force were presented as the sum of the forces associated with the dispersion of the workpiece material and the friction of the petal grains against the workpiece, which carry out cutting and plastic deformation. We took into account the peculiarities of the location of abrasive grains along the depth of the working layer of the petals made of a grinding belt [10]. The geometric shape of the grains was presented in the form of a truncated cone, the diameter of the small circle of which is equal to the size of the blunt area formed on the grain during wear.

The radial $P_{y}$ and tangent $P_{z}$ components of the grinding force were determined as:

$$
P_{y}=P_{y r 1}+P_{y r 2}+P_{y d 1}+P_{y d 2} ; \quad P_{z}=P_{z r 1}+P_{z r 2}+P_{z d 1}+P_{z d 2}
$$

where $P_{y r 1}$ and $P_{z r 1}, P_{y r 2}$ and $P_{z r 2}$ - radial (index $y$ ) and tangential (z) components of the force due to microcutting of the workpiece material and friction of the cutting grains of the petals on the workpiece, $\mathrm{N} ; P_{y d 1}$ and $P_{z d 1}, P_{y d 2}$ and $P_{z d 2}-$ components of the force due to plastic deformation of the workpiece material and friction of plastically deforming grains on the workpiece, $\mathrm{N}$.

Cutting grains are located at a distance $y$ from the conditional outer surface of the petal: $0 \leq y \leq y(l)$, where $y(l)$ - a function describing the dependence of the depth $y$ of occurrence of AG on the length $l$ of its contact with the workpiece. In determining $P_{y r 1}$ multiple integration is used to sum the microcutting forces from the individual AG.

$$
P_{y r 1}=\int_{0}^{l_{k}}\left[\int_{0}^{y(l)} P_{y r 1 i} \cdot n_{\mathrm{zk} 1} \cdot c_{k} \cdot d y\right] \cdot d l,
$$

where $l_{k}$ - arc length of contact of the cutting grain with the workpiece, $\mathrm{m} ; P_{y r 1 i}-$ microcutting force by individual $\mathrm{AG}, \mathrm{N} ; n_{z k 1}$ - the number of $\mathrm{AG}$ on the surface of the petal in the section by a plane parallel to the axis of the circle, $1 / \mathrm{m} ;\left(n_{z k 1}=Z_{0} \cdot H\right.$, where $Z_{0}$ - number of AG on a single lobe area, $1 / \mathrm{m}^{2} ; H$ - the size of the workpiece surface to be machined in a direction parallel to the axis of the circle, $\mathrm{m}) ; c_{k}$ - coefficient characterizing the distribution of grain depths in the working volume of the petal, $1 / \mathrm{m}$.

$$
y(l)=h_{u r}+a_{z}(l)
$$

where $h_{u r}$ - deterioration of grain performing microcutting, $\mathrm{M} ; a_{z}(l)$ - function describing the change in the depth of penetration of AG into the workpiece material along the contact length $l$.

Forces to cut the workpiece material with a single grain:

$$
P_{y r 1 i}=\tau_{s} \cdot F_{m} \cdot k_{y}
$$

where $\tau_{\mathrm{s}}$ - shear strain during microcutting by grain of the workpiece material, $\mathrm{Pa} ; F_{m}$ - area of the workpiece metal removed by a single grain, $\mathrm{m}^{2} ; k_{y}$ - coefficient [5]. 


$$
F_{m}=\left(2 h_{u r}+a_{i r}\right) \cdot a_{i r} \cdot \operatorname{tg} \gamma
$$

where $a_{i r}$ - the depth of penetration into the workpiece of the i-burning AG, $\mathrm{m}$ [5]; $\gamma$ - half of the angle at the apex AG, degree.

To calculate $a_{z}(l)$ used the dependence [11]:

$$
a_{z}(l)=\frac{V_{s} \cdot \sin \alpha_{\max } \cdot l_{m} \cdot l}{\pi \cdot D_{k} \cdot n_{k} \cdot l_{k}},
$$

where $l_{m}$ - average distance between the cutting grains of the petal, m; $V_{s}$ - flap wheel longitudinal feed speed, $\mathrm{m} / \mathrm{min} ; D_{k}$ - circle diameter, $\mathrm{m} ; n_{k}-$ wheel speed, 1 / min; $\alpha_{\max }-$ angle, degree [11].

The result is the dependence:

$$
\begin{aligned}
& P_{y r 1}=k_{y} \cdot \tau_{s} \cdot B_{p} \cdot\left(2 \cdot h_{u r}^{2} \cdot a_{k r} \cdot l_{k}+\left(6 \cdot h_{u r} \cdot a_{k r}+7 \cdot h_{u}^{2}+a_{k r}{ }^{2}\right) A \cdot \frac{l_{k}^{2}}{2}+h_{u r} \cdot a_{k r}{ }^{2} \cdot l_{k}+\right. \\
& \left.+\left(h_{u r}+a_{k r}\right) \cdot h_{u r}^{2} \cdot l_{k}+\frac{h_{u r}^{3} \cdot l_{k}}{3}\right),
\end{aligned}
$$

where $a_{k r}$ - critical depth of microcutting, when exceeding which the grain performs microcutting, $\mathrm{m}$;

$$
A=\frac{V_{s} \cdot \sin \alpha_{\max } \cdot l_{m}}{\pi \cdot D_{k} \cdot n_{k} \cdot l_{k}} ; \quad B_{\mathrm{p}}=\operatorname{tg} \gamma \cdot n_{z k 1} \cdot c_{k} .
$$

To calculate $P_{y \mathrm{p} 2}$ dependences were obtained:

$$
P_{y r 2}=\frac{\pi \cdot l_{2 r}^{2} \cdot k_{y} \cdot n_{z k 1} \cdot c_{k}}{12 \cdot \mu_{s}} \cdot \tau_{s} \cdot\left(h_{u r} \cdot l_{k}+\frac{A \cdot l_{k}^{2}}{2}\right),
$$

where $l_{2 r}$ - the size of the blunt area on the cutting grain, $\mathrm{m} ; \mu_{\mathrm{s}}$ - internal friction coefficient.

To calculate the radial component of the force from plastically deforming grains associated with deformation of the workpiece material $\left(P_{y d 1}\right)$ and friction of plastically deforming grains against the workpiece $\left(P_{y d 2}\right)$, dependences were obtained.

$$
\begin{aligned}
& P_{y d 1}=\frac{c \cdot \sigma_{t s} \cdot n_{z k 1} \cdot c_{k} \cdot \operatorname{tg} \gamma}{2}\left(\sin \gamma+\mu_{0} \cdot \cos \gamma\right) \cdot l_{k 1} \cdot a_{k r} \cdot\left(\frac{a_{k r}^{2}}{3}-h_{u d}^{2}\right) ; \\
& P_{y d 2}=c \cdot \sigma_{t s} \cdot \frac{k_{y} \cdot \pi \cdot l_{2 d}^{2} \cdot n_{z k 1} \cdot c_{k}}{12} \cdot a_{k r} \cdot l_{k 1},
\end{aligned}
$$

where $l_{k 1}$ - length of the trajectory of contact of the plastically deforming grain of the tape with the workpiece, $\mathrm{m} ; c$ - coefficient $[5,11] ; \sigma_{t s}$ - yield strength of the workpiece material at the 
temperature and speed of grinding, $\mathrm{Pa} ; l_{2 d}$ - the size of the blunting area on the plastically deforming grain, $\mathrm{m} ; h_{u d}$ - deterioration of grains carrying out plastic deformation, $\mathrm{m} ; \mu_{0}-$ coefficient of friction of grain on the workpiece.

To calculate the tangential component of the force $P_{z}$ dependences were obtained:

$$
\begin{aligned}
& P_{z r 1}=\frac{k_{z}}{k_{y}} P_{y r 1} ; \quad P_{z r 2}=\mu_{0} \cdot P_{y r 2} ; \\
& P_{z d 1}=B_{d} \cdot\left(\mu_{0} \cdot \sin \gamma+\cos \gamma\right) \cdot l_{k 1} \cdot a_{k r} \cdot\left(\frac{a_{k r}^{2}}{3}-h_{u d}^{2}\right) ; P_{z d 2}=\mu_{0} \cdot c \cdot \sigma_{\mathrm{T}} \cdot D_{d} \cdot a_{k r} \cdot l_{k 1},
\end{aligned}
$$

where $k_{z}$ - coefficient [5].

When calculating the average distance between the cutting grains of the petal, it was assumed that the number of AG in contact with the workpiece increases linearly with increasing depth of their occurrence in the working volume of the petal. The number of AG on a single area of a petal made of a grinding belt was calculated from the dependence [10].

When calculating the shear stresses averaged over the shear plane, which determine the microcutting force by the petal grains, we used the data of the work [2].

A numerical simulation of the grinding force and their components during the processing of workpieces made of aluminum alloy D16 with a flap wheel with an outer diameter of $D_{k}=150$ $\mathrm{mm}$, abrasive grain material $-25 \mathrm{~A}$; grit P60; wheel height $H=0,02 \mathrm{~m}$. Grinding mode: working speed of the petal circle $V_{k}=26 \mathrm{~m} / \mathrm{s}$; longitudinal feed speed $V_{s}=1,2$ and $3 \mathrm{~m} / \mathrm{min}$; grinding depth $t=0,0048 \mathrm{~mm}$.

\section{Experimental research}

Experimental studies were carried out on a surface grinding machine under the same conditions under which the numerical simulation of forces was performed. The components of the grinding force were measured with an UDM-100 dynamometer, the signal from which was fed to the TDA amplifier and then to the 16/16 - SIGMA / USB ADC. Used the ZetLabStudio software.

The results of numerical modeling and experimental research are presented in Table 1.

Table 1. Results of numerical modeling and experimental study of the grinding force component

$$
P_{y}
$$

\begin{tabular}{|l|c|c|}
\hline Longitudinal feed speed $V_{s}, \mathrm{~m} / \mathrm{min}$ & 1,2 & 3 \\
\hline Calculated value $P_{y}, \mathrm{~N}$ & 24,8 & 26,3 \\
\hline Experimental value $P_{y}, \mathrm{~N}$ & 26,9 & 30,0 \\
\hline
\end{tabular}

With an increase in the longitudinal feed rate from 1,2 to $3 \mathrm{~m} / \mathrm{min}$, the calculated and experimental values of the force Py increased by 6 and 12\%, respectively.

The maximum discrepancy between the calculated and experimental values does not exceed $14 \%$, which indicates the possibility of using the obtained analytical dependences for calculating the grinding forces with flap wheels. 


\section{Key findings}

Summing up the forces associated with the dispersion and friction of the cutting and plastically deforming petal grains on the workpiece, we obtained analytical dependences for calculating the grinding forces with flap wheels,

Numerical modeling and experimental studies have confirmed the possibility of using the obtained analytical dependences for calculating the grinding forces with flap wheels.

With an increase in the longitudinal feed rate 2,5 times, the component $P_{y}$ of the grinding force increases by $12 \%$.

\section{Acknowledgments}

This work was supported by the Russian Foundation for Basic Research and the Government of the Ulyanovsk Region within the framework of the scientific project No. 19-48-730002.

\section{References}

[1] Gdalevich A.I., Zhitnitskiy S.I., Khrychev V.I. etc. Polishing of parts with flap wheels. - M .: Mechanical Engineering, 1980. - 80 p.

[2] Korchak S.N. Productivity of the process of grinding steel parts. - M.: Mechanical Engineering, 1974. - 280 p.

[3] Filimonov L.N. Resistance of grinding wheels. - L .: Mechanical engineering. Leningrad branch, 1973. - $136 \mathrm{p}$.

[4] Ostrovsky V.I. Theoretical foundations of the grinding process. - L.: Publishing house of Leningrad State University, 1981. - 144 p.

[5] Efimov V.V. Model of the grinding process using lubricating coolant. - Saratov: Publishing house Sarat. un-that. - 1992. - 132 p.

[6] T. Yu, H. Su, J. Dai, W. Zhou Grinding Force and Specific Grinding Energy in Process of Grinding SiC with Single Grit. Nanjing Hangkong Hangtian Daxue Xuebao. Journal of Nanjing University of Aeronautics and Astronautics. - 2018. - 50(1):120 -125.

[7] Deguo Li, Jinyuan Tang, Haifeng Chen, Wen Shao. Study on grinding force model in ultrasonic vibration-assisted grinding of alloy structural steel. The International Journal of Advanced Manufacturing Technology. - 2019. - 101(9-12)

[8] Unyanin A.N., Khusainov A.S. The ultrasonic grinding process temperature field study // MATEC Web of Conferences. - 2017. C. 01011. SJR - 0,166.

https://doi.org/10.1051/matecconf/201712901011

[9] Dubrovsky P. V. Grinding of titanium alloys with flap wheels. - Ulyanovsk: U1STU, 2000. $100 \mathrm{p}$.

[10] Verezub V.N. Sanding with abrasive belts. - M.: Mechanical Engineering, 1972. - 103 p.

[11] Khudobin L.V., Unyanin A.N. Minimization of contamination of grinding wheels. Ulyanovsk: U1STU, 2007. - 298 p. 